\title{
Philosophie et théologie face aux émotions
}

\section{Bernard Forthomme}

\section{La théologie est-elle plus proche de la tragédie que la philosophie?}

Les plus anciennes acceptions du terme théologie proclament leur complicité avec les narrations mythiques et leurs articulations rituelles, jusqu'à ce que la philosophie donne à la théologie une autre allure. Démarche où la recherche n'est plus celle, surchargée de pathétique et d'illusions, d'une représentation factice de l'origine et du spectacle de la fin, de cette mise en scène d'une prime vie sexuelle et de la mort ultime. La fascination par les cris premiers qui président à la naissance ou à la reproduction de la vie, et par les râles qui gouvernent l'agonie en sus de la jalousie voire de la malignité divine, une telle fascination n'a plus cours dans le champ de la philosophie. Néanmoins, c'est bien la philosophie qui nomme d'abord la théologie comme usage critique et allégorique de la mythologie et du théâtre tragique-dramaturgie qui tamise en réalité le 
mythe et le rationalise déjà. Ensuite, c'est encore la philosophie qui assigne le champ poétique (homérique) à la différence face au domaine philosophique. C'est même la Métaphysique d'Aristote qui conçoit la connaissance théologique comme science théorique (comme philosophie première) après les mathématiques et la physique. Mieux: avec le courant stoïcien, la théologie devient vraiment une discipline philosophique, une "physique", alors qu'avec le néoplatonisme, la théologie s'apparente plus intimement encore à la philosophie.

La pensée chrétienne hésite face à cet enracinement mythologique, rituel et philosophique de la théologie antique. D'autant que le mythe homérique conserve, jusque dans sa traduction dramatique ou tragique, l'idée de fond d'une coupure radicale entre le divin et l'humain, même si les traits humains du divin, favorisent très tôt l'évolution d'une théologie de la substance première ou du principe vers une théologie de la parole faite chair, expérience, irrégularité, société, image, génération sexuée, temps, histoire et mortalité. De manière assez révélatrice, il faut attendre l'émergence de l'hégémonie politique chrétienne pour que le terme de théologie devienne une notion chrétienne, mais encore très éloignée de ce que l'on conçoit généralement depuis Abélard et à sa suite surtout, avec le développement de la pensée juridique et de sa forme critique, sans oublier l'influence des commentaires d'Aristote et de son concept de science, même si la distinction aristotélicienne de sciences théoriques est déjà assimilée depuis Boèce.

La théologie reste proche de la mythologie, de la liturgie et de la configuration par l'expérience sanctorale, dans la mesure où elle désigne encore le discours de Moïse, des prophètes, les écrits de Paul_-et donc la prédication universelle, avec son usage rhétorique des émotions - l'évangile de Jean ou carrément toutes les Ecritures néo-testamentaires, ainsi de façon inattendue chez le fameux Denys; même si, chez lui, 
coexistent des distinctions internes différenciant la théologie affirmative de la théologie apophatique ou mystique. Soulignons d'ailleurs que la tradition latine continue longtemps d'user d'un langage qui trahit cette proximité entre théologie et Ecritures: sacra scriptura, sacra pagina ou doctrina sacra (tirée des Ecritures) sont des syntagmes usuels jusqu'à ce que la scolastique baroque systématise décisivement la théologie, en ruinant le genre du commentaire suivi, le style sentenciaire.

La théologie baroque inclut certes une forme aiguë du pathos, mais elle prétend surplomber les Ecritures par la jouissance du divin, de ses images, l'importance prioritaire de qui parle ou légifere, en usant des textes, inspirés ou non. Cette tendance trouve une compensation dans la réduction de la théologie à la morale voire à l'affectivité-jusqu'à la rébellion possible et justifiée (même si elle est évitée par charité)-ou à l'expérience mystique. Il est vrai que la contestation opérée par la théologie dramatique vise précisément une forme de systématisation qui devient un monologue, élimine le côté dialogal de la théologie présent depuis la pensée patristique jusque dans la scolastique des réponses et des questions. Mais c'est une rupture également avec la philosophie ancienne, fameuse précisément pour le développement du dialogue.

Tant que la Bible demeure la référence majeure, en lien étroit avec la liturgie (ce qu'on désigne souvent par Bible et son exégèse n'est souvent qu'un ensemble d'extraits liturgiques ou homilétiques), la prédication, la vie contemplative ou l'expérience des saints, le pathos du point de vue de Dieu-la Jalousie, la Colère, la Joie divines-s'inscrit encore profondément au cœur de la théologie. La compréhension du terme évolue en fonction des grands débats concernant la relation trinitaire et les natures du Christ. La théologie devient de plus en plus une affirmation de la vérité comprise comme vérité commune face à l'hétérodoxie. La théologie se pense alors elle-même comme une connaissance (trinitaire) distinguée 
d'une pratique (économie salvifique) et d'une expérience contemplative. La théologie mystique opère également un déplacement vers les trois voies spirituelles, plus proches en un sens de la philosophie que de la Bible. Au lieu de commencer par la voie purgative ou la catharsis du sensible, l'exégèse biblique des quatre sens tient à conserver toute la densité initiale de la signification sensible, corporelle ou temporellecritiquant ainsi une approche des seules quiddités ou des natures intemporelles, sans l'événement du temps et de la faute contagieuse qui marque le cheminement spirituel. C'est seulement ensuite que cette initiation dramatique se déploie dans les sens spirituels, l'ascèse tropologique et la finalité unitive. En rompant avec l'adéquation entre les trois voies et le tempo des exercices spirituels scolastiques, la lignée ignatienne manifeste déjà une redramatisation de la vie spirituelle, avant même de relever son usage réglé de la scénographie imaginative et du rôle axial de la répétition.

Certes, si l'on s'interroge sur la nature de la théologie, ses vecteurs platoniciens, son appétit majeur pour la vérité qui ne s'en laisse pas conter, se soumettant la fable des origines et des fins dernières, la dimension poétique ou mythique (le beau) et la forme rituelle ou politique (le bon), de tels vecteurs font croire que la complicité entre théologie et philosophie est initiale. Or il pourrait n'en être rien, surtout si l'on considère le fond liturgique, spirituel et prédicationnel de la théologie, jamais totalement disparu, mais réhabilité radicalement aujourd'hui comme célébration communautaire du mystère divin et de son événement, jusque dans son annonciation et son usage des émotions ou de la rhétorique qu'elle implique. La parenté entre le logos théologique et le pathos dramatique, tragique en particulier (à cause du sérieux biblique), semble s'imposer avec autant de force.

Mais cette parenté n'est pas une simple retrouvaille, comme si les tendances logiques ou magistérielles avaient 
gouverné depuis l'époque médiévale. En réalité, c'est bien plutôt la lignée augustinienne ou anselmienne, et la lignée affective inaugurée par la théologie cistercienne, victorine et franciscaine transformée en théologie de la volonté, qui a fini par percer, et se retrouve dominante à l'époque moderne, aussi bien par l'affirmation de la volonté infinie cartésienne que par la prédestination luthérienne et calvinienne, jusqu'à l'affirmation de la volonté chez Schopenhauer (en cela bien éloigné du bouddhisme) et Nietzsche (en cela bien éloigné de la pensée grecque ancienne et pas seulement socratique), mais encore dans la lignée juive, marxienne, bergsonienne ou freudienne. Ce puissant courant se trouve recueilli, même fort différemment, dans la philosophie française contemporaine, chez Michel Henry comme chez Gilles Deleuze-même si, chez ces penseurs, le primat revient à la force spinoziste plutôt qu'à la volonté proprement dite dont la consistance n'est là que logique. La liberté sartrienne, par contre, se montre plus fidèle à la volonté cartésienne. Il demeure que la théologie comme science spéculative est en déclin durable.

Quant à la volonté augustinienne, elle ne semble pas si hostile aux émotions qu'il semblerait à première vue dans sa censure de la passion involontaire. La mise en cause ne porte-telle pas avant tout sur une passion qui exprimerait un refus de se laisser émouvoir par Dieu, même si l'option analytique ou naturaliste consistant à faire de la raison une simple expression des émotions, serait-elle sublimée, se voit révoquée? L'essentiel de la critique porte toutefois sur le refus de consentir à la jouissance d'un Dieu vivant et vrai (jouissance distincte de son usage intéressé). Ainsi donc, c'est bien plutôt le souci apologétique tendant à réagir face au romantisme finissant, au dolorisme comme à l'activisme pastoral et missionnaire, comme au scientisme croissant, qui a conduit à un renouveau de la théologie déductive et déterministe-la liberté elle-même y semble déduite de l'évidence de la finalité, de la vérité ou de 
l'évidence du bien fini et infini--pour redonner, croyait-on, un niveau scientifique à la sagesse théologique.

Comment expliquer alors ce sentiment animant la théologie dramtique de renouer avec des influx patristiques vu le déclin de la théologie spéculative et l'éphémère idéal du "déterminisme"? C'est sans doute parce que la théologie de la volonté elle-même, assimilant l'impact biblique serait-ce dans une dynamique néoplatonicienne (ainsi le primat du Bien sur la dualité noétique chez Plotin ou celui-là même de la volonté entériné par le Fons vitae d'un penseur juif comme Ibn Gabirol, à l'époque de Pierre Damien) mais encore stoïcienne, s'est traduite en termes de science déductive, même s'il s'agit avant tout d'une science pratique comme on le voit dans le courant scotiste jusqu'à l'optique cartésienne, alors que l'augustinisme bonaventurien est éclipsé durant six siècles jusqu'à sa résurgence institutionnelle ou éditoriale. Néanmoins, l'autoimplication du théologien, si importante dans l'orientation bonaventurienne face à la manière logique-même si la conception de la béatitude suivant le Commentaire des Sentences place l'amour et la connaissance dans un rapport équilibré--, une telle orientation perpétue et transforme la priorité de la volonté qui justifie une science plus pratique que spéculative, ouvrant la voix à la critique kantienne, sinon rousseauiste, du rationalisme de la théologie philosophique (substance spinoziste, monade leibnizienne, même si la monade leibnizienne n'est pas étrangère au principe d'individuation scotiste, via la scolastique baroque). La théodramatique de $\mathrm{H}$. $U$. von Balthasar elle-même prend en relais, suivant un vecteur ignatien, la précellence moderne de la volonté sur l'intellect, même si la volonté se donne à entendre comme beau vouloir ou se laisse configurer par la forme héroïque ou sanctorale.

Toujours est-il que la philosophie critique la tragédie, et très tôt, dès avant Platon, dans les Catharmes d'Empédocle. Ce dernier s'en prend non seulement à la tragédie, mais peut-être 
d'abord à sa racine liturgique, même si elle n'est pas la seule et si le cérémonial du théâtre n'a pas la fonction du rite au sens fort, sacrificiel en l'occurrence, qu'il s'agisse de l'homme ou de l'animal; le sacrifice reçu ici comme affect social fondamental apaisant l'angoisse magico-religieuse au lieu de produire l'angoisse de la volonté agonique, serait-ce pour la réguler. La philosophie conteste le sacrifice humain, le sacrifice tragique d'Iphigénie ou d'Antigone, même si Antigone n'est pas indemne de culpabilité et si l'amour ou la haine sont présentés comme des forces d'organisation des éléments physiques. Il reste que le logos philosophique explicitement distinct de la poésie-celui de Platon ou d'Aristote-exclut l'émotion du divin ou de la connaissance qui se connaît elle-même, même si la poétique réhabilite les passions tragiques au plan a-moral qui leur est propre. Malgré l'usage de la tragédie par le philosophe comme instrument, organe d'une mise en relief de thèses sur la volonté finalisée, les relations, leurs jugements sociaux ou divins, il faut attendre la philosophie romantique pour voir la tragédie servir à une dramatisation profonde de la pensée. Avec parfois une réduction de la tragédie à une simple illustration d'une thèse ou d'une problématique abstraite comme celle d'une opposition entre l'individu privé et l'Etat.

À vrai dire, si la théologie se rapproche tellement de la tragédie, n'est-ce pas d'abord sous l'influence de la philosophie, au-delà de l'usage pédagogique platonicien, de la poétique d'Aristote ou même de la dialectique hégélienne qui enrôle intimement le chour tragique et la figure d'Antigone dans son élaboration profonde, vérifiant et donnant un relief saisissant à la théorie du progrès conflictuel de la conscience (ich bin beide Kämpfende und der Kampf selbst)? N'est-ce pas aussi l'existentialisme qui, en contestant le primat de l'essence au profit de l'acte individuel, s'est non seulement ouvert au théâtre en configurant intimement l'esthétique ou le dépassement de la connaissance par des types dramaturgiques (Don Juan, 


\section{BERNARD FOR'I'HOMME}

Faust), mais est devenu acteur dans le champ théâtral (songeons à Albert Camus, Jean-Paul Sartre ou à Gabriel Marcel)? La théologie qui prétend trouver une source majeure dans la tragédie, grecque de manière exemplaire, n'est-elle pas tributaire de ce tournant philosophique né après la Révolution française?

Il est vrai que le courant kierkegaardien-pris en relais à sa manière par la pensée heideggerienne de l'être en étroite correspondance avec l'épreuve de l'angoisse et de la chutegarde une inspiration théologique flagrante (mais déjà la phénoménologie husserlienne ne se laisse-t-elle pas inspirer explicitement par Augustin, alors que le concept ontologique de chair chez Merleau-Ponty doit beaucoup à la théologie chrétienne et française du corps?). Néanmoins, aurait-on songé à prendre la forme tragique comme modèle de la forme théologique, si Nietzsche n'avait osé contester ce qui, pour lui, constitue le moralisme socratique et tout le platonisme (dans lequel il inscrit erronément le christianisme et sa forme idéaliste)? D'autant plus que cette critique de la figure de Socrate est déjà préparée par la critique métaphysique hégélienne: la scission socratique entre l'innocence du prévenu et le refus de contester les lois de la cité, réduisant le drame à une question de victime innocente rejetant la procédure, est considéré comme un symptôme d'une conscience qui n'est pas encore retournée dans la patrie de l'Unité. Bref, l'évolution de la théologie dans un refus progressif du système conceptuel et scolastique se montre bien plus dépendante de la critique philosophique que certains théologiens ne veulent bien le reconnaitre.

En outre, l'affirmation de la primauté de la tragédie dans la formation de l'esprit théologique au détriment de la philosophie, n'est-elle pas dépendante d'un préjugé humaniste, entendons littéraire? Ce qui conduit à poser cette question: la théologie est-elle plus proche de la littérature, et donc d'une forme esthétique, que d'une recherche philosophique de la vérité? 
La théologie ne serait-elle vraiment que le fruit d'une convergence de la littérature biblique et de la tragédie? L'expérience des protagonistes tragiques serait alors plus proche de celle des héros bibliques, des martyres, de l'expérience des saints, que de la convergence, pourtant si constante et attestée, entre Platon, le néo-platonisme et la pensée patristique, sans oublier la convergence avec Aristote, mais commenté à partir d'un point de vue monothéiste, par Avicenne ou Averroès, Thomas d'Aquin, Duns Scot, jusqu'à la scolastique baroque d'un Suarez?

Et pourtant, il reste alors à se confronter à trois difficultés majeures. Tout d'abord, si l'expérience des saints favorise le primat de la volonté, comme on le constate de manière flagrante dans la recherche de la pauvreté volontaire chez François d'Assise, il n'est pas moins remarquable de constater que la salutation de la sagesse (regina sapientia) précède chez lui la salutation de la pauvreté et de la charité, du moins dans la Salutatio virtutum. Comme si l'on ne pouvait avoir accès à la volonté pure sans la sagesse. Cet équilibre est maintenu dans la pensée bonaventurienne où amour et connaissance s'embrassent, mais également dans la conceptualisation scotiste de la volonté qui récuse explicitement que la cause totale de l'acte volontaire soit la volonté subjective, son intensité, faute de quoi l'on ne saurait distinguer entre l'amour d'une mouche et celui de l'infini. Certes, il est vrai que le motif baroque de la mouche trahit à quel point non seulement le singulier s'exalte dans la tendance nominaliste moderne, mais à quel point la différence d'objet voulu ne se marque plus que par la différence d'intensité. La logique fétichiste ne reconnaît d'ailleurs à l'objet quelconque qu'une valeur transitoire face à l'intensité même du désir qui l'anime. Mais l'émotion implique une forme de connaissance de la différence. Elle sait bien que l'objet fétichiste, en l'occurrence, est dérisoire, et ne mérite pas que l'on s'y attarde pour lui-même, mais le désir ne peut faire autrement que d'y passer. 
L'émotion participe étroitement de la connaissance, serait-ce comme connaissance repoussée en dernier ressort dans ce qu'elle révèle de futile ou de factice.

Ensuite, autre difficulté, relevons que la justification des passions comme motions-distinctes de la disposition moraleet leur valorisation face à un certain encratisme chrétien, à certaines tendances platoniciennes et stoïciennes de la patristique, est un acquis de la poétique aristotélicienne, même s'il peut également servir à un naturalisme, notamment médical ou psychiatrique, cherchant à réduire, par exemple, la tristesse de la dépression à un comportement, à une motion ralentie (soluble pharmacologiquement), un réfrènement dont l'affect ne serait que le commentaire ou l'expression subjective périphérique. Certes, vivre selon les passions et vivre selon la raison, voilà qui reste très distinct. En ce sens, la dimension biblique de la théologie chrétienne inscrit plus franchement les passions, la jalousie préférentielle, l'indignation, la colère et l'amour, au cour de la vie divine et prophétique. Plus précisément encore, la dimension charnelle de la parole portée par un peuple singulier, formé par la préférence jalouse du Dieu unique, favorise l'inscription des émotions les plus concrètes au sein de la parole de vie et de vérité. Ce qui ne fera que s'amplifier par l'annonciation et la manifestation unique de cette parole chez un homme mis à part. Le logos devient inséparable de la chair, même si l'affirmation de la résurrection du corps se passe dans l'expérience de la présence comme en celle de la disparition. Il reste que l'idéal d'autonomie et de contrôle des émotions demeure orienté par le dynamisme de la métanö̈a, du bouleversement de l'esprit inspiré par la charité.

Toutefois, si la tradition biblique inscrit généreusement les émotions au sein du logos, et si l'on reconnaît la présence de certains éléments tragiques dans quelques fragments bibliques, nous devons constater que la Bible ignore la forme même de la tragédie. Et s'il n'y a pas de forme tragique, c'est 
sans doute pour de fortes raisons sur lesquelles on ne peut s'attarder ici, mais qui ne peuvent se réduire ni à une question purement cultuelle (naissance supposée et contestable du théâtre dans la liturgie païenne), à un fait culturel (absence générale de thêâtre dans la culture sémitique, y compris en islâm sunnite), ni à l'opposition entre une vision esthétique d'un côté, et une option éthique de l'autre. Sans parler de l'instance critique ou la relativisation-la mise en perspectiveque constitue la mise en scène du monde divin par le théâtre: le Dieu d'Israël paraît à la fois trop différent, mais aussi trop proche (conscience prophétique), pour tolérer ou subir pareille manipulation et un tel distanciement.

De plus, peut-on sérieusement penser la naissance de la tragédie sans tenir compte des liens étroits qui nouent cette forme dramatique à l'émergence de la philosophie, à la rhétorique, à la politique (l'isonomie démocratique), à la pensée juridique-impliquant une vérité surgie du débat public contradictoire, et non plus d'un secret préalable ou inaccessible, ni d'une vérité éternelle-comme à l'athlétisme? Il existe une correspondance profonde entre ces différents domaines où les extrêmes se rencontrent.

La célébration de la victoire olympique (nous dirions aujourd'hui sportive) de l'individu est non seulement une opération de vérité, d'anamnèse luttant contre l'oubli, mais également contre la jalousie envieuse: la rivalité légitime des protagonistes ne doit pas empêcher de louer l'adversaire, comme Pindare l'affirme explicitement. Louange qui a des effets pratiques dans la mesure où elle féconde d'autres actes prestigieux. La tragédie est aussi la mise en jeu d'une figure: non un type général, mais un nom, un particulier prélevé sur l'histoire pour entraîner le spectateur dans le dynamisme de l'action. Il est vrai que le rapprochement avec le chœur tragique s'impose d'autant plus que la célébration de la gloire du vainqueur antique n'est précisément pas seulement celle d'un 
individu ni d'une griffe, mais de la Cité à laquelle il appartient, et même de son pays, de la terre où il est né. On est loin de l'exaltation volontariste du torero face à la masse participant à une corrida.

L'athlétisme grec permet de faire concourir musicalement le corps et l'esprit, l'ami et l'ennemi dans la vitesse ou l'agilité de l'exercice et la paix du jeu (contrairement aux jeux romains)-même s'il lui manque cette universalité concrìte de la paix qui sera pensée et articulée par Paul de Tarse, à l'échelle de l'Empire (où les jeux sont, malgré leur violence, un facteur de civilisation et d'unification des colonies), dans la mission apostolique, la course chrétienne vers la victoire irrévocable, toujours suivant l'image paulinienne. La victoire de l'athlète du Christ n'étant jamais achevée tant que le temps donné à l'universalisation de la paix ne trouve pas son point d'application et d'accomplissement dans la conversion achevée du peuple particulier d'où est issu le Christ, formé par la jalousie préférentielle. De même, la tragédie, son coefficient d'accélération des orages, d'articulation des impasses, permet de déceler la part de vérité propre aux antagonistes: les lois de Créon, leur urgence politique, ne sont pas moins nécessaires que la loi universelle du soin des morts dont se réclame la désobéissance d'Antigone avec une impatience digne du fruit de l'inceste. La tragédie persiste, car elle provient de ce que les lois du ciel et de la terre, comme celle des vivants et des morts, ne trouvent pas leur unité.

Par contre, la tragédie permet aussi de rendre visible l'invisible, d'avoir accès au secret des conseils divins et de contracter une épopée entière, d'abréger, d'accélérer les événements qui se déroulent in vivo sur plusieurs décennies, voir sur plusieurs vies. La tragédie est animée par un double mouvement de scission et de fermeture ou d'immanence. Scission entre le divin et l'humain, mais immanence de l'acte: tout se joue sur scène, dans l'événement actuel, sous nos yeux. 
Néanmoins, la tragédie ne parvient à parfaire cette présence, cette contraction, que sous la forme du spasme, de la souffrance, des éclats de colère ou des émotions amoureuses cruelles. L'abrégé de la colère miséricordieuse et de l'amour de Dieu se manifeste non seulement dans les contractions de la gésine et du trépas, mais dans le transitus fulgurant de celui qui a été préféré pour résumer Dieu.

Remarquons, malgré tout, la volonté polémique d'une certaine forme de théologie qui s'annexe la tragédie: son désir de jouer sur tous les tableaux de la tragédie contre la philosophie. D'où sa prolixité et ses risques de vague conceptuel. Si la philosophie affirme la proximité de l'humain et du divin dans la participation ou l'unité de l'être, on lui oppose la transcendance et la fracture tragique. Si la philosophie affirme la différence entre l'être et le phénomène, récusant une densité mythique, on lui oppose la présence liturgique et l'effet sacramentel de l'instant tragique, serait-ce avec des accents kierkegaardiens, comme acuité accrue de la souffrance. Ce qui rejoint le primat de la densité ontologique propre à la tendance johannique et apocalyptique.

Finalement, cette densité n'est découverte que dans la personne médiatrice immédiate: l'être messianique en qui l'être est le paraître. Or c'est bien là que le radicalisme johannique et la violence apocalyptique manifestent leurs limites internes. Simples figures de la densité et de la violence divines. Sous le système sacrificiel, émergent l'animal égorgé, individuel, l'homme cloué, singulier insubstituable, comme un Dieu ambivalent, sa cruelle miséricorde.

Malgré l'affirmation de la densité ou de l'immanence tragique, il convient de rappeler l'importance de la généalogie des maux: le rappel du caractère intraitable d'Edipe apparait chez sa fille Antigone, comme une des conséquences vénéneuses de l'inceste. Ce raide attachement à son propre point de vue, cette colère fermée sur elle-même ou sur un seul 
aspect de la loi, et cet aveuglement sur l'amour des autres, voilà qui n'est pas éclairé seulement dans l'événement actuel. Tout n'est donc pas présent. Une déhiscence subsiste au sein de l'acte.

L'inceste est également lié à l'absence de soin du corps trépassé, à la privation de sépulture. L'inceste parental mine non seulement la civilisation, mais l'hominisation: la privation de sépulture fait passer de l'homme à l'animal, et d'abord aux crocs des animaux. Le ventre animal devient la tombe de l'homme. On trouve plus tard ce rapprochement entre inceste et privation de sépulture dans la légende de Sainte Dympna (ca 1250), cette princesse irlandaise qui résiste aux assiduités de son père jusqu'à son exécution et sa privation de sépulture. Mais contrairement à la tragédie grecque, sa sainteté se manifeste déjà du seul fait que les animaux ne la mangent pas. Au lieu de passer à l'animal, l'inceste entravé fait passer à l'ange. Mais ange ou démon, l'inceste oblige l'humanité à se déporter loin d'elle-même. Même la résistance réussie à l'inceste expose à cette séparation, à ce passage de l'humanité à la sainteté.

Mais revenons au silence biblique touchant le théâtre. Le malaise biblique face à l'action théâtrale est certainement lié au fait que la mise en scène tragique expose ainsi dangereusement le divin à l'esprit critique. Le monde mythique réalisé sous les yeux des spectateurs, accroît son humanisation et son articulation dialogale, susceptible d'antistrophes et de polémiques. Le monde de la prière devient dialogue. Et c'est ce dialogue--rompant le face à face fascinant entre le protagoniste et le spectateur-qui contribue à l'émergence de l'esprit critique de la philosophie, du droit et du politique, de sa forme démocratique.

Or s'il n'y a pas de forme tragique dans la Bible, malgré Job et tant de moments tragiques, il faut bien dire que, dans le même temps, il n'y a pas plus de forme philosophique ni proprement démocratique présente en elle, pas plus que de 
jeux olympiques. Ni théâtre ni jeux, ni philosophie, ni politique. $\mathrm{Ni}$ politique des jeux, ni philosophie politique.

Certes, la Bible contient le lyrisme des psaumes et une conception ambivalente du politique. $\mathrm{A}$ la fois comme espace désiré (émergence d'une exigence d'autonomie) et comme pratique impossible (soumission de la forme de l'Etat au vouloir divin), ainsi qu'on le surprend dans l'élection de Saül et dans son échec. Même l'impunité de David sera sans suite, vu la déroute finale du régime salomonien, la déchirure du royaume qui prive la maison de David, et pour longtemps, de la souveraineté sur tout le peuple.

La différence et l'identité dans l'Obéissance au divin: c'est cela qui ferait se rapprocher fondamentalement la tragédie de la théologie. La volonté soumise? Le pathos serait au fond une manière de rencontrer la volonté soumise. Mais qu'en estil de la colère et du don chez Euripide? Qu'en est-il de Médée et d'Alceste? Où est l'obéissance? Ne s'agit-il pas plutôt du pur amour, de l'autonomie, de l'auto-motricité de la volonté, même s'il s'agit d'une volonté blessée?

La logique de la colère reste distincte de la violence naturelle, de l'agressivité. Elle est un appel à la loi, à une issue plus rapide, à une justice dont la vitesse est accrue (ainsi qu'on le surprend de manière frappante lors de la Révolution française, jusque dans l'usage de la guillotine). Colère distincte en cela de la transgression qui prend plaisir dans le viol de la loi, ou plus profondément encore, comme chez Augustin, qui jouit du vol lui-même, de l'acte de voler, de la volonté partagée de voler-ce même Augustin par qui la volonté stoïcienne s'inscrit vraiment comme un axe majeur de la théologie, en devenant volonté confessante ou pathétique, inscrite dans un puissant lyrisme, celui de la joie propre à la louange!

Il reste que le plaisir augustinien du vol n'est pas solipsiste: il se révèle plus fort et même seulement possible dans la complicité, une volonté conjuguée de voler en groupe. Peu 
importe ce qui est volé. C'est l'affirmation de soi dans le vol, couplée au plaisir de nuire. Mais le plaisir est encore pensé comme une conséquence d'une volonté qui connaît le bien sans choisir positivement le mal, suivant la logique de la liberté d'indifférence chez Occam, de la volonté perverse chez le Malin génie cartésien, ou celle du mal radical chez Kant.

Toujours est-il que la tragédie permet de configurer l'idéal d'une volonté dont le dynamisme ne vient pas de son objet ou de sa finalité, mais d'elle-même. Volonté comme pur amourpur au sens de définalisé-car celui pour lequel meurt Alceste n'en vaut guère la peine. Il n'y a là aucune finalité première. Il ne s'agit pas seulement de mourir pour ni même de mourir avec, mais suivant les cas tragiques repris par Platon, de mourir en plus ou par dessus... C'est moins l'obéissance au destin ou à la destinée que l'acte volontaire transformant la contrainte de mourir.

Au fond, ce qui est reproché injustement à la philosophie, c'est de refuser une immanence sous forme de souffrance et de sacrifice humain. C'est de refuser ce principe sacrificiel de l'obéissance. Cela que la tragédie accepte, comme la Bible et la Théologie.

Dieu autorise la cruauté: on passe d'Adam le végétarien à Noé le carnivore " terreur des animaux ». Cruauté encore de la Pâque juive: massacre des uns (fils aînés et agneaux), salut des autres. Là est le nœud au-delà des ruptures: le sacrifice d'Isaac, de la fille de Jephté ou d'Iphigénie et, avant tout, le droit affirmé de YHWH sur le premier-né (Michée 6,7), et qui se réaffirme lors du massacre des premiers-nés d'Egypte.

Mais la croix du juste crucifié, nouvel agneau pascal, remémore et dénonce cette cruauté rémanente dans la perception ancienne de Dieu, le massacre du déluge, celui des premiers-nés d'Egypte ou des prémices (animales, pas seulement végétales), tous les anathèmes. 
L'image biblique de Dieu est souvent ambivalente. Dieu parait plus d'une fois comme le roi David, son « fils »: a-moral! Malgré ses fautes plus fortes que celles de Saül, le fils préféré reste l'objet de la promesse d'une maison inébranlable et se voit maintenu dans les voies du Seigneur. Son impunité est celle des héros comme Abraham qui livre sa femme, comme Isaac qui trompe Pharaon ou l'étranger Abimélek, comme Jacob, comme Joseph, comme Jephté, Samson....

La Pâque chrétienne rappelle l'innocence des agneaux (des animaux et des enfants, la cruauté de Dieu) au sein de l'Agneau immolé. L'agneau, c'est le fils aîné de l'Egypte tué injustement pour notre délivrance. Le coût de ma liberté est un crime, même si je ne suis pas coupable. La croix rappelle que derrière l'agneau, il y a l'homme mis à mort injustement. Pour rien, gratuitement. La dimension radicalement critique de la croix met en cause la représentation de l'agneau qui masquerait la cruauté.

Cruauté encore et toujours comme celle des lois touchant la répudiation. Cruauté au sein de la loi et pas seulement dans une loi réalisée sans esprit, à la lettre. L'esprit de la loi peut être cruel. Ézéchiel s'en méfie dans un passage fameux $(20,25)$. La loi retient la violence (en elle)! Elle n'en affranchit pas de manière décisive. Cruauté des lois qui font mourir, serait-ce martyrs. Cruauté du Livre des témoins d'Israël et des Apocalypses. Une théologie qui fait fond sur le genre apocalyptique penche naturellement du côté de la violence et de la cruauté qui est retenue dans la révélation: contenue comme foyer et contenue comme régulation de son débridement. La dimension liturgique sous-jacente à la vision apocalyptique ne doit pas faire oublier sa mise en scène de l'Agneau immolé. Ce n'est précisément pas un agneau, mais un être humain qui est crucifié injustement, dans un acte de violence crue, excluant toute justification liturgique et sacrificielle. Il y a un artifice à rapporter la mort cruciale au 
rite de la Pâque. Néanmoins, c'est alors que le rite pascal luimême est dévoilé dans sa profondeur: la cruauté de la mise à mort de l'animal et de l'homme.

Lorsque Tertullien critique les spectacles du $\mathrm{III}^{\mathrm{e}}$ siècle comme leur généalogie, et enjoint à ceux qui ne peuvent se passer de la vue du sang frais (la vie), de se contenter du sang du Christ, il tente d'inscrire l'envie de voir le commencement et la fin, le sexe et la mort-impuissants à se montrer comme tels-envie qui s'exprimait dans le spectacle romain, au sein même de la dramatique cruciale. Seulement là, en ce champ, les passions peuvent se voir sans le voile du mythe, nez à nez ! Émotion crue qui somme la volonté de consentir ou de révoquer, serait-ce en choisissant positivement le mal reconnu comme un mal. Alors le tragique de la vie, même s' il est surmonté par la résurrection, s'expose à la forme théâtrale du drame.

Ce n'est pas un hasard si la forme théâtrale a vu son réaménagement explicite en Occident non d'abord par dramatisation des dialogues hagiographiques, mais au sein de la liturgie pascale: non d'abord comme liturgie de la Passion ni comme liturgie magico-religieuse (servant l'accès aux forces divines), ni même simplement comme rite confirmant une vérité connue, mais au contraire comme trope, ce qui brise l'unité du rite-même si la liturgie se sert de la forme théâtrale pour sa visée spécifique, fonder ou confirmer. Il ne faut pas confondre le cérémonial théâtral (susceptible de prendre distance avec le fondement, de changer de rythme, d'accroitre l'Effroi) et la fonction spécifique du rite, l'harmonisation, la solution de l'anxiété. La plus ancienne reformulation connue en Occident depuis la disparition des théâtres sous l'impact barbare et la subsistance de spectacles dont certains devaient sans doute comporter des moments d'art théâtral, une telle refiguration explicite apparaitt sous forme d'une interrogation, d'une question de recherche qui concerne tout homme: Qui chercheq-vous? 
(Quem quaeritis?) -ainsi qu'on le voit au Xe siècle dans le fameux Introit de Pâques selon saint Ethelwold, évêque de Winchester dans sa Regularis Concordia (datée de 965/75). Le drame se tient alors dans l'orientation de la recherche entreprise avec émotion par les femmes venues embaumer le corps du précieux défunt, comme par tout être confronté au trépas d'un être cher, plus cher que lui-même: un mort ou un vivant? Et quel vivant?

\section{L'émotion tragique et la parole équivoque}

L'équivoque ici n'est pas d'abord un défaut mais ce qui plaît à l'esprit théologique dans la mesure où il ne clôture pas l'homme sur sa position, même si l'équivoque dramatique reste rigide comme ses masques d'acteurs, et ne peut se comparer jusqu'au bout à l'ambivalence de Dieu que nous venons d'évoquer, car elle évolue, se transfigure et se démasque. Il demeure que les positions exclusives de Créon et d'Antigone n'éliminent pas le chant du Chœur tragique et son discours diplö̈de. Une brèche reste ouverte pour effectuer la demande tragique de salut.

Le tragique articulé dans la tragédie ancienne n'est pas l'absurde. Non seulement parce que le tragique implique le sens de la volonté scandalisée, résistante, mais de l'acte juste réfrénant le destin. Tout le tragique d'Antigone vient de ce que la justesse de la position de la sœur qui désire enterrer son frère, même celui qui menaçait la cité, ne peut s'imposer absolument, sans tenit compte de la justesse de la position de son oncle qui entend condamner celui qui menace l'Etat, car la vie politique n'est pas le seul fruit d'un arbitraire humain, même s'il est aussi une contingence. Il n'y a tyrannie qu'à partir du moment où le roi prétend énoncer seul la parole véritable. Comme dit le fils de Créon: l'Etat (plus précisément la Cité: polis) n'est plus l'Etat, dès qu'il appartient à un seul homme (andros énos; v. 734). Mais cette sentence vaut aussi pour 
Antigone: elle ne doit pas se cabrer sûre de son propre point de vue, dans une rigidité incestueuse, sans égard pour sa sœur ni pour celui qui l'aime et qui y perdra la vie.

Néanmoins, ce que Créon ne comprend pas-et ne peut aisément comprendre car le frère d'Antigone s'est rebellé-et ce que le Ménexìne de Platon va mettre en relief, c'est que le travail de deuil est nécessaire à la gloire de la Cité. Certes, les éloges funèbres sont souvent une illusion, une forme de charme ou d'ensorcellement de nos âmes (goèteuousin hèmôn tas psychas; 235a) qui donne l'impression d'appartenir à une cité héroïque. Le discours de Platon s'engage néanmoins dans l'éloge et inclus l'importance traditionnellement relevée de l'autochtonie, de la terre privilégiée et de sa mise en valeur par les ancêtres, mais également la défense de la liberté qui ne redoute pas la guerre contre soi-même, la guerre civile. Il reste précisément que Socrate, dans le Gorgias, préfère précisément lutter contre sa propre Cité pour la rendre meilleure comme un médecin et non comme un flatteur (521 a). L'éloge de la Cité n'est pas accepté sans critique. Et ce qui est mis en accusation, c'est moins l'émotion comme telle que le manque de spontanéité, et la production rhétorique des émotions avec un rapport plus étroit à l'illusion, au plaisir d'écouter son éloge en écoutant celui du mort, qu'à la vérité elle-même. Le deuil qui manque d'émotion et de spontanéité, celui qui crée l'émotion par l'institution sociale et l'habileté rhétorique vise facilement à se plaire lui-même et non la vérité du deuil. La philosophie, une fois de plus, rompt moins avec le tragique, l'émotion du deuil, qu'avec l'absence de tragique des lieux communs articulés par les éloges funèbres, car cette absence signe le mensonge et l'ineffectivité du labeur de deuil.

Quant au discours d'Antigone, il vise également à protéger la cité, ses parents, ses ancêtres. Or précisément, la tragédie censure l'autognôsos, car Antigone ne prend conseil que d'elle-même-ce qui trahit certes la généalogie, celle de l'inceste 
toutefois, les étreintes incestueuses de ses parents: koimìmata autogennèta (v. 859). La tragédie censure aussi bien la prétention de Créon à ne voir de sagesse que dans son seul discours. Son fils, approuvé par le chœur, lui rappelle d'ailleurs que la sagesse ne règne pas seulement dans ses seuls discours, mais dans ceux des autres: le langage et l'esprit, il ne les possèdent pas seuls (v. 705).

Néanmoins, l'autognose de la victime de Créon semble transformer le destin de la mort et la décision légale de son anticipation, par une volonté lucide de mourir en faveur de son frère unique, irremplaçable, conformément à une loi non-écrite, plus importante que la loi édictée. S'il s'était agi d'un mari ou d'un enfant qui aurait pu avoir des successeurs, le choix aurait été différent. Alceste ira plus loin qu'Antigone en allant jusqu'à mourir à la place de son mari qui ne l'aime d'ailleurs que modérément.

Face à l'autognose qui entrave l'ouverture théologique comme la dialectique philosophique, le chœur conserve ce sens de l'équilibre tragique entre les positions antagonistes de la nièce et de l'oncle, soumis au même destin, mais sans être en mesure de trouver une véritable concordance entre les protagonistes. L'émotion tragique--laissée à elle-même, sans autre différence que le destin anonyme-fissure toujours par avance une telle concordance. Cette position, comme on le sait, est typique du chœur tragique grec: « Prince, il te convient d'écouter ce qu'il y a de juste dans ce discours (il est raisonnable de te laisser enseigner: se te mathein); et toi aussi, écoute ton père, car vous avez sagement parlé tous les deux (eirètai gar eu dipla; v. 722) ». Le discours diplö̈de approuve donc la nécessité du rite funéraire, mais également la volonté du roi de refuser l'anarchie. Son excès est de vouloir que l'on sacrifie tout à la volonté paternelle (gnômès patrôias; v. 637). Ce qui le conduit à croire que l'obéissance sauve la plupart des corps (peitharchia sôzei ta polla sômata; v. 673). Il identifie l'ordre de la Cité à celle 
du cosmos: à l'harmonie universelle !

Mais il n'y a pas que le cosmos, sa beauté. Il y a aussi l'anarchie de la mort, à laquelle précisément le rite d'ensevelissement entend mettre bon ordre et beauté. À la guerre de la mort s'oppose la paix du rite; à l'informe du cadavre, sa forme; à l'animalité dangereuse pour la nature humaine (dont la figure est exposée aux becs et griffes de l'animalité), la forme humaine confirmée. Autrement se déchaînent des forces inquiétantes, des bruits sinistres, des vols funèbres, des pressentiments atroces auxquels le devin fait droit en interprétant les signes et les énigmes. C'est manquer de sagesse que de ne pas y prêter l'oreille. C'est manquer un précieux remède à la situation inextricable: la colère enfonce dans un monde plus stupéfiant, moins rationnel que le déchiffrement mantique et philosophique; par ce biais, la mantique rejoint la médecine entendue comme ce qui déchiffre des signes, les moments favorables, sans s'en tenir à une logique qui ignorerait les fluctuations de la clinique, l'ambivalence des symptômes et la complexité des fièvres. Ce n'est pas que la politique doive se soumettre à l'obscure prophétie: mais la philosophie peut chercher à déchiffrer le sens des signes comme la mantique de Tirésias.

Déjà Platon réconciliait, dans son Phèdre, la critique socratique et le rôle de la fureur érotique mise en parallèle avec la fureur télestique (rituelle), mantique et poétique ("folies" étroitement liées). On doit d'ailleurs voir dans la volonté d'Antigone une fureur ou une manie proprement télestique. La fureur érotique habite plutôt son époux, alors que la fureur mantique anime Tirésias. La folie poétique semble se garder dans les chants du chœur. D'ailleurs, Antigone le lui reproche: " on se rit de moi (gelômai). Pourquoi m'insulter avant ma mort?» (v. 838 ). Puis le chœur se reprend en arguant de l'hérédité fatale, des suites de l'inceste qui ruine la vie familiale, les liens filiaux, conjugaux, linguistiques et rituels, comme la vie de Cité. 
Le chœur ne fait pas que guérir ou départager sagement: il réveille aussi les anciennes douleurs; c'est la mémoire vivante, la mémoire participative.

Toutefois, la réduction basse de la fureur mantique manifestée par le devin à l'appât du gain, cette avidité si commune dans le monde de la divination, aveugle le politique qui ne s'appuie que sur ses seules ressources légales. Les émotions seront plus fortes que la crainte de la force politique (kratos), bien qu'elle soit reconnue comme aussi valable que la piété envers les corps défunts (v. 867 ss.). Les émotions contenues du chœur vont se déchaîner chez Antigone, son époux, le fils du roi et chez l'épouse de ce roi, lui qui parle seul et ne règne plus dès lors que sur une cité déserte, désertée par ses habitants!

Soulignons ici à quel point le discours diploïde du chour tragique empêche la conception aristotélicienne du possible. Il n'y a pas continuum physique unilatéral entre la puissance et l'acte comme réalisation de cette unique puissance; acte réduit à n'accomplir que cette puissance physique-la forme sise dans la matière. La tragédie ne s'oppose pas ici à la philosophie: elle prépare plutôt une autre perception du possible et une émancipation de la volonté par rapport à la physis: deux choses peuvent être possibles en même temps.

La doctrine ultérieure de la volonté ira plus loin en pensant une double puissance pour un seul acte (sans effacer par cet acte l'autre possible), et non plus seulement pour deux actes, même si chacun reste alors dans l'unique bien-dire. Ici nous n'avons pas encore la liberté d'indifférence.

Sur le plan tragique, c'est l'émotion qui permet de conserver l'équilibre choral, serait-ce l'émotion du corps social. En effet, c'est l'émotion mutuelle, l'ouverture de la source des larmes qui permet au chœur de s'affranchir des lois de Créon, mais à un plan différent: non pas en soutenant ouvertement d'autres lois, ou en s'alignant directement sur la volonté 
autonome d'Antigone l'indépendante (autonomos; v. 818), ce qui l'expose à une mort rapide-contre le désir de Créon qui lui prépare une mort lente, et sans que personne ne la touche, de sorte que personne ne puisse être crédité de sa disparition. Que chante le chœur? Ceci: « Moi-même en ce moment, rebelle aux lois de Créon (litt.: je suis emporté hors des lois: phéromai exô thesmôn; 7.798 s.), je ne puis retenir la source de mes larmes, à la vue d'Antigone marchant vers la demeure où dorment les mortels ».

Autrement dit, l'équilibre n'est maintenu qu'à partir du déséquilibre de l'émotion. Mais c'est une émotion chorale, un " je » schématique, et non psychologique. Emotion qui permet de rétablir une forme de lien entre des positions en apparence antithétiques: même les lois politiques ou célestes ne sont pas moindres que les dispositions de la mort. Erôs n'est donc pas opposé à la pulsion de mort. Il peut conserver et détruire: c'est une force invincible (v. 778) qui concerne la Cité comme la campagne, les mortels comme les immortels. Erôs, cette fureur, peut emporter le sens des justes vers l'injustice et le crime, susciter une querelle fatale entre parents, au-delà des lois. Erôs est rapproché de l'émotion du chœur qui excède les lois. Il est toutefois terrible de considérer cette force emportée vers la vie comme vers la mort, voire le dépassement de la vie immanente.

\section{L'émotion comique}

Le comique (à distinguer du rire déclenché, effusif), dont la formc thćâtralc précède probablement celle du tragiquc, nous fait rejoindre ce dont tout théâtre participe: simultanément une adhésion plus grande aux saillies de la vie sociale, aux aléas de l'existence, de ses plaisirs, de ses revers, de ses violences-la matière théâtrale se prélève autant sur le spectacle du cirque, de la fête foraine que du mime, du cabaret, du ring ou du 
combat de coqs-et une fonction spécifique de mise à distance, de séparation, qui est propre au théâtre, comique ou non. C'est sans doute pourquoi il n'y a pas forme de théâtre dans la tragédie ordinaire, les rites des familles, des tribunaux, les parlements, des milieux d'affaires ou des planifications socialistes, des insolences ludiques adolescentes ou des protocoles sportifs dont l'importance sociale reste d'ailleurs adjacente par rapport aux rites de fondation anciens. Toutefois, le théâtre comique ne semble pas animé foncièrement par l'espérance de modifier réellement les figures de l'existence dont il dessine les contours comme les codes qu'il inverse pour le plus grand bonheur des spectateurs. Il reste que la philosophie des Lumières s'est souvent senti l'alliée des émotions comiques pour éclairer la pensée, élucider les forces politiques et religieuses, pour modifier les mœurs, les rapports familiaux et judiciaires, tout comme l'expression critique de la mémoire, des connaissances historiques ou des taxinomies. Ceci s'accompagnant souvent d'un discrédit radical de la Bible-plus que de Dieu, voire d'un christianisme éthiqueaccompagné d'une incompréhension proportionnée.

Peut-être l'obéissance prophétique comme l'impunité des héros politiques mais aussi patriarcaux, empêchent-elles véritablement l'émergence biblique du théâtre tragique aussi bien que comique: le sérieux biblique est dominant, quoi qu'on en dise pour faire le malin. Il n'y a pas moyen de prendre réellement distance représentative par rapport à la destinée. La loi divine se montre à la fois trop proche-inscrite non seulement dans la pierre mais dans les cours-et trop intangible, sauf viol des consciences. Il n'y a pas moyen de se détendre ! sinon par les cris de guerre, l'orgie de la victoire, la joie du salut. Par la naissance d'un fils, d'un premier-né, qui rompt la fatalité de la stérilité ou le malheur inexorable. Joie de briser les appropriations définitives de l'être, les enclos qui entravent sa spontanéité et son repartage. Joie de briser les 
incarcérations, les surdités, les paralysies et les aveuglements. Joie de voir jaillir l'accomplissement d'une promesse longtemps désirée.

Or l'émotion théâtrale opère à la fois un distanciement par rapport à une certaine gravité de la vie, et un rapprochement de ce qui se donne inaccessible, antérieur, futur jamais assez, lointain, secret, supérieur, céleste ou invisible, intangible. La force dramatique rend visible et tangible, unifie déjà le ciel et la terre, mais aussi les enfers, le divin, l'humain et l'infernal, les anges, les démons, les hommes, les héros et les lâches, les aristocrates et les gens du peuple, les animaux, les rois et les sujets, les dédales de l'intériorité et les plus hauts faits, sans y parvenir complètement-sans toutefois que cela apparaisse comme la téléologie première d'un rite magico-religieux, avec son efficience inductrice d'appartenance communautaire et de solution d'angoisse.

Le rire implique bien une certaine légèreté face à la pesanteur du sacré, même si sa mise en forme théâtrale enrôle sa ritualité sociale, voire sa dimension magique, sinon initiatique dans certaines cultures. Certes, il ne faut pas confondre le comique, son essence, et l'événement même du rire, qu'il soit d'ailleurs forcé ou spontané; même forcé, le rire peut poursuivre sur sa lancée et devenir naturel. Quant au comique, il ne déclenche pas nécessairement le rire, mais parfois une blessure, l'indifférence affectée, le dépit courroucé ou la hargne. Lorsqu'on n'aime plus quelqu'un, ses émotions peuvent nous paraître comiques. Et le rire, de son côté, n'exprime pas seulement le flux de la vie face aux raideurs habituelles, mais la relation déterminée, l'antipathie ou la joie de rencontrer un être cher, un enfant, un animal familier, une idée enthousiasmante, un mot qui sonne juste. Le rire peut se produire lorsqu'on découvre un nouvel aspect d'une question, un concept approprié, une expression verbale heureuse, un vers, une rime, une tonalité, une rapidité d'élocution. Le rire 
est aussi une accélération de l'existence, aisément liée à la vitesse d'une pensée ou de la parole. Le rire ne peut donc se ramener à la crise des médiations sociales, intellectuelles ou verbales. Il exprime aussi et réalise une qualité de médiation, une intensité d'articulation, une vitesse du langage.

Il reste que la philosophie des Lumières a bien compris tout le parti qu'elle pouvait tirer de la force du comique et/ou du rire pour modifier le destin de la pensée, de la conversation, de l'écriture et des mours. Toutefois, la fonction critique du rire est bien présente dans la Bible, non seulement dans les récits touchant la génération patriarcale, mais surtout dans le contexte prophétique lorsque les prophètes ou les écrits qui s'en inspirent exercent leur humour face aux idoles. La philosophie voltairienne teste tributaire de l'usage prophétique du sarcasme, même s'il est finalement retourné contre la Bible et les pratiques idolâtres des Chrétiens.

Toutefois, s'il est vrai que le rire se distancie d'une certaine pesanteur sacrale, ce n'est pas qu'il soit radicalement incompétent dans le domaine du sacré; ce n'est pas un hasard s'il se laisse ritualiser, lui et de ses connotations génésiques, dans le quotidien, la pratique sociale, mais également dans des rites initiatiques. Autre manière d'épouser la réalité, mais à fleur de peau, susceptible de s'en détacher comme un papillon d'une fleur. C'est une superficialité comme sensibilité épidermique, vitesse supérieure grâce à laquelle en même temps le poids de la réalité nourricière se tient à distance et se mesure, tout en se laissant féconder. L'os et la chair sont tragiques. Non que la chair soit triste, mais elle est grave. L'humour érotique ou "politique" est justement une manière permanente d'alléger cette gravité, le drame de l'origine, de la vie, des liens, des pouvoirs et des gloires comme de la mortalité. Il implique également le ressort de l'angoisse. C'est aussi une modification de l'angoisse et pas une simple affirmation de la vie, même si le rire est d'abord une exultation de la vie. Plus précisément: 
c'est une affirmation de la vie qui contient l'angoisse de la mort, jusqu'à ce qu'elle se lâche, éclate, se débride soudain. Le rire révèle la gravité de la destinée dans la mesure même où elle en affranchit. Et il est probable que le rire a pris forme théâtrale avant la forme tragique.

En un sens, la comédie est plus proche de l'émotion et de la volonté que la tragédie. Car la noblesse du sujet tragique se déporte parfois de son atmosphère religieuse délivrant une grandeur morale ou interne, vers les pensées nobles des protagonistes. Ici la comédie épouse mieux le vouloir déterminé de vivre ou l'axiome implicite, que l'effort anonyme du destin. La volonté s'entiche d'objets plus particuliers que la conservation de soi. Certes, elle se fait plus étroitement complice d'une réalité sociale-les anciens sujets de rire vieillissent très vite et mal-et en ce sens, elle est plus désacralisée que sa forme tragique. Mais la volonté veut gaspiller sa vitalité, au plus proche de la vie que des concepts objectifs ou formels, médiateurs de la vérité objective toujours distante.

La comédie épouse au plus près le dynamisme de la vie, même si la volonté reste indépendante par rapport à l'inclination ou la disposition vitale. Il reste un lien à la sacralité de la vie en deçà de la complaisance sociale. Est-ce une fluidité qui s'apparaît à elle-même avec des traits caricaturaux, des mimes plus typés, sans psychologie fine, comme dans les contes populaires? On pourrait dire ici que c'est plutôt l'inverse.

La comédie est aussi un exercice d'indépendance, de spontanéité, de vagabondage dans les lieux, les temps, les personnages de l'histoire, les doctrines, les affects les plus pervers, par rapport à la rigidité de la vie. La comédie accélère tellement la vie, qu'elle en trouble les images closes, les caractères, les méandres, les plis. La comédie défroisse, déplie la vie. La comédie déride! La méchanceté s'y métamorphose, elle n'est jamais elle-même jusqu'au bout. Elle est dévoyée de sa logique, désamorcée aussitôt naissante et réapparaissante. 
La vie la plus vivante est encore trop rigide pour la comédie inspirée, car trop complice du mourir.

La comédie est plus actuelle ou plus rapide, plus véloce que la tragédie. D'ailleurs, nous l'avons relevé, la tragédie se passe essentiellement à basse vitesse, sur le mode d'un réfrènement du destin, même si cela ne l'entrave pas de manière décisive. L'accélération comique trouve son point d'encrage, moins dans le sujet léger que dans la mise en scène, l'acte des réalisateurs. La volonté comme liberté s'y affiche. Une désinvolture par rapport au destin, sinon à la finalité de son existence y rend le jeu gratuit. Le théâtre lui-même est dupliqué. Non plus seulement comme scène d'illusions ou de folie, de rêve, mais comme lieu de dépassement du théâtre dans sa facticité et son élément factice.

L'essentiel devient l'émotion, l'intensité de l'émotion, de l'éclat de rire, plus que l'objet transitoire, fugace, sans importance intrinsèque. Ce qui peut menacer la densité ontologique de l'immanence, alors qu'elle semblait pourtant le plan majeur de la comédie. Ici ce n'est plus sous le jour de la densité sacramentelle comme dans la tragédie, mais de la densité qui devient manipulable, percée à jour, sphère fissurée, fêlée par le rire. La rigidité paranoïaque, celle de la tragédie, celle d'un Saül par exemple, devient celle d'un automate comique. Ce qui peut conduire à l'inconsistance du sujet et à un déterminisme de l'acte, un karma, remplaçant le déterminisme du destin ou de l'Idée. C'est là le foyer d'une convergence portant nom de tragi-comique. Tragi-comédie recommandée par un V. Hugo notamment, mais en plaçant comme Kant le comique du côté du corps, et le tragique du côté de l'âme. Néanmoins, la volonté individuelle reste présente dans la tragédie comme dans la comédie, autrement cela passerait sous nos yeux sans intérêt!

Mais ne faut-il pas faire un choix dans cette oscillation? Un choix face à une conception indifférentiste ne s'impose-t- 
il pas? Même si, dans les Ecritures, le même événement peutêtre vu tantôt sous l'angle comique, tantôt plus tragique, le sérieux domine. Même si ce sérieux est limité par le sentiment d'impunité des personnages majeurs, sujet de la promesse. Comme si la promesse enlevait la force de tout le tragique, dès le début. Ce qui rend la distinction, la synthèse et l'indifférence même du tragique et du comique à peu près impossible ou secondaire.

Toutefois, le comique qui brise les formes anciennes, déstabilise le prestige des beautés indiscutables, des définitions arrêtées, des rôles qui incarcèrent le soi au lieu de lui trouver un jeu plus adéquat à son action personnelle, un tel comique se pense volontiers comme répétition; ce qui n'exclut pas un durcissement de la vie au sens où il en ferait la caricature et la gaieté. Il y aurait le tragique et la reprise comique. Toutefois, l'ambivalence de la répétition interdit d'identifier la reprise à une farce. La répétition peut guérir, délivrer des formes fascinantes, en dégager la facticité sinon le factice-l'analyse psychologique joue sans cesse de ce ressort-l'arbitraire du mal, mais également aggraver les maux, intensifier le tragique.

Critique des mythes, de l'harmonie cosmique, prenant le courant de la volonté ouverte sur un avenir non-répétitif, la répétition comique n'est pas une simple imitation. Elle produit une autre perception de la volonté menacée par les impasses de la vie, les choix impossibles, la paralysie morale. Toutefois, au sein de l'expérience antique, la pire impasse conserve un sens, une issue, voire une possible réconciliation. Le comique n'est donc pas un simple dépassement de l'absurde. On l'a dit et répété, le tragique n'est pas l'absurde. Toujours demeure un sens de l'injustice, celui d'une forme de volonté agonique qui empêche de réduire le personnage à un vide ou à une vaine agitation, voire au simple grotesque.

L'importance de l'éclat de rire a crû dans le théâtre de la cruauté, car il marque la présence du corps et de la volonté, 
face à la parole. C'est le refus de l'harmonie analogique, de la concordance des noms qui, en fait, cache un acosmisme et une indigence ontologique, une supériorité de la négation sur la similitude. Autrement dit, l'émergence du cri défait le privilège des discours ou des répliques qui sonnent creux, et finalement comme une trompeuse communication. Les cris rompent les formes rigides du beau et du laid, mais c'est pour affirmer le mystère de la vie et de ses tourbillons, pour mieux rendre compte aussi de la puissance du corps à souffrir l'inorganique, l'infini sans lieu (zone érogène) ni topique, l'intolérable désir, son effraction.

Certes, le tragique pur comme épreuve du destin n'est pas proprement chrétien. Mais des situations concrètes peuvent être vécues sur le mode tragique. Mieux, le rôle tragique peut être attribué comme supposition impossible pour rendre compte au plan théorique de ces situations tragiques qui ne sont pas des affirmations dogmatiques sur le destin. Cela rencontre l'expérience mystique, avec ses descentes au purgatoire ou aux enfers, in vivo, jusqu'à l'anonymat de la nature humaine défigurée, sans personne, voire jusqu'aux racines élémentaires de la physis. Enfer plus fort encore si l'on est juste que si l'on est coupable. Situation en théorie impossible, mais éprouvée dans l'expérience religieuse comme dans la colère chalamovienne. Même l'épreuve de la noirceur, d'un Malin génie cartésien ou d'un Mal radical kantien, d'une volonté qui veut positivement le mal, peut être expérimentée comme mise à l'épreuve de la plus pure charité, distraite de son objet comme cause finale.

Toutefois, la théologie n'est pas seulement une sagesse spirituelle repérée dans le sensible jusqu'à la contemplation de l'expression intra-divine en passant par une folie cruciale (pas simple crise, car modalité inouie de la même sagesse), mais une science. Entendons: ce qui reste dans le champ du logos, participe à la communauté des savoirs, et surmonte les ex- 
pressions mythiques locales-serait-ce par impératif de prédication universelle. Ce qui fait se croiser intimement la logique philosophique et la rationalité théologique, même si un chiasme se dessine bientôt entre ces deux exercices de la pensée.

Pour pouvoir s'inculturer, assimiler au mieux les mythes ou les rites locaux, les cultures particulières et les différences émotionnelles ou autres liées à la sexuation, la théologie doit se libérer sans cesse du mythe et du rite appartenant à des cultures mortes, comme des exclusives ou des interprétations liées à la domination contingente d'un sexe sur l'autre, d'une partialité émotionnelle sur une autre. Mais la théologie nourrie de parole biblique, de son expérience émotive complexe, ne peut s'en tenir à une logique purement anhistorique ou un savoir, serait-ce un savoir-faire, finalement élitiste: elle est plus intimement exposée encore que la philosophie à de nombreux paramètres hétérogènes, aux témoins et aux témoignages, aux expériences spirituelles différenciées, aux sources manuscrites, à la philologie et aux sciences historiques ou exégétiques, aux pratiques sociales ou communicatives, ainsi qu'aux services de gouvernement, régulateurs ou incitatifs.

Il est sans doute légitime d'enraciner cette science théologique dans la sagesse spirituelle et la folie cruciale qui la justifie, dans la liturgie et la prédication, mais c'est de la nécessité de communiquer ce qui excède la communication naïve, arrogante ou aveugle, ce qui empiète sur le circuit fermé, privé d'esprit et d'émotion de recherche, que provient la nécessité de la forme scientifique de la théologie. L'expression théologique n'est pas réductible à un magistère épiscopal ou liturgique, à un discours universitaire ou pédagogique (celui des manuels), pas plus qu'à l'expérience mystique ou féminine, sans parler du despotisme culturel, ethnique, social ou dévotionnel. La justification de son logos et des émotions qui l'animent face aux autres discours 
PHILOSOPHIE E'I'I'HÉOLOGIE FACE AUX ÉMO'ITONS

ou comme mise à l'épreuve interne de soi, de sa vérité, de ses articulations plus conformes au logos qui l'inspire, est aussi une exigence souveraine de la vie spirituelle en quête d'affranchissement et de vérité vive.

Centre Sèrres, Paris 